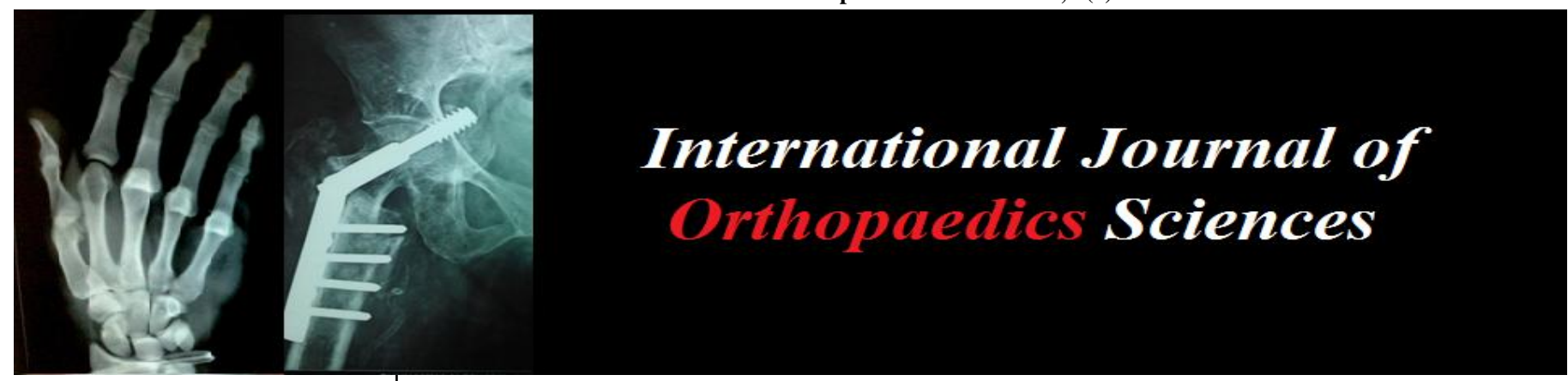

ISSN: $2395-1958$

IJOS 2017; 3(4): 548-552

(C) 2017 IJOS

www.orthopaper.com

Received: 15-08-2017

Accepted: 16-09-2017

Dr. Swaroop Das

M.S. Ortho, Senior Resident Department of Orthopedics Dr V.M. Govt. Medical College, Solapur Maharashtra, India

Dr. Harshal V Kalambe

M.S. Ortho, Senior Resident,

Dept. of orthopaedics BJMC and

Sasoon Hospital, Pune,

Maharashtra, India

Dr Sunil P Handralmath M.S. Ortho, DNB Ortho Professor and Head Of Department Dept. of Orthopedics Dr. V.M. Govt. Medical College, Solapur,

Maharashtra, India
Correspondence

Dr. Harshal V Kalambe

M.S. Ortho, Senior Resident,

Dept. of orthopaedics BJMC and

Sasoon Hospital, Pune,

Maharashtra, India

\section{Comparative study of unstable intertrochanteric fracture treatment by trochanteric femoral nail versus hip hemiarthroplasty}

\section{Dr. Swaroop Das, Dr. Harshal V Kalambe and Dr. Sunil P Handralmath}

DOI: https://doi.org/10.22271/ortho.2017.v3.i4h.75

Abstract

The management of unstable intertrochanteric fractures in elderly is challenging because of difficult anatomical reduction, poor bone quality, and sometimes a need to protect the fracture from stresses of weight bearing and the need for early mobilization. Internal fixation in these cases usually involves prolonged bed rest or limited ambulation, to prevent implant failure. This might result in higher chances of complications like pulmonary embolism, deep vein thrombosis, pneumonia, and decubitus ulcer. The purpose of this study to compare the results of trochanteric fixation nailing (TFN) and hip hemiarthroplasty in unstable intertrochanteric femur fractures in elderly patients.

Out of 50 patients with unstable intertrochanteric fractures 25 patients were treated with primary cemented bipolar hemiarthroplasty and 25 were treated with trochanteric femoral nail. Follow up was done post operatively at 1 months, 3 months, 6 months. At each follow up visit patients were evaluated radiologically and clinically. Functional outcome was evaluated by Harris hip score. Out of 25 patients operated by hemiarthroplsty, 3 patients had excellent results and 12 patients had good results whereas 9 patients had fair and one patient had poor results. Out of 25 patients operated with TFN, one had excellent results and 8 patients had good results whereas 10 patients had fair and 6 had poor results. Two hemiarthroplasty patients and one TFN patient developed superficial infection whereas one dislocation was also observed in hemiarthroplasty patient. Screw cut out from head was seen in one TFN patient and one TFN patient also developed pulmonary embolism.

The cornerstone of management of such fractures is early surgery, followed by mobilization. Hence Cemented bipolar hemiarthroplasty offers a modality of treatment that provides adequate fixation and early mobilization in these patients thus preventing postoperative complications such as pressure sores, chest infection and DVT, as compared to TFN where there was more chance of complication due to prolonged immobilization.

Keywords: Unstable intertrochanteric femoral fractures, cemented bipolar hemiarthroplasty, trochanteric femoral nail (TFN), harris hip score (HHS)

\section{Introduction}

Intertrochanteric fractures are those occurring in the region extending from the extracapsular basilar neck region to the region along the lesser trochanter. Due to an increasing life span, the incidence of these fractures is increasing in elderly ${ }^{[1]}$. They are associated with high rates of morbidity and mortality due to the need for prolonged immobilization, although results are improved with use of recent modalities of internal fixation ${ }^{[2]}$. Approximately $15 \%$ to $20 \%$ of patients die within 1 year of fracture ${ }^{[3,4]}$.

Intertrochanteric fractures in younger individuals are usually the result of high energy injury, such as a motor vehicle accident and other traumatic injuries. But Ninety percent of intertrochanteric fracture in the elderly results from a trivial fall.

Early and adequate fixation is very important in elderly patients so as to mobilize them at earliest and prevent complications of recumbency like bedsores, deep vein thrombosis and respiratory infections.

Among the intertrochanteric fractures, the unstable type fractures pose more difficulty in treatment as well as prognosis. Osteoporosis and difficult anatomical reduction are one of the most important factors leading to unsatisfactory results in these fractures ${ }^{[5-7]}$. 
The goal of treatment is to achieve stable anatomical reduction, rigid fixation, and early mobilization of patient and prevent deformity at hip.

Hemiarthroplasty for intertrochanteric fractures has been described as early as 1973. It is now being considered as a primary treatment for comminuted unstable type of IT fracture in elderly on the grounds that it allows early mobilization and full weight bearing.

Recently popular modality is fourth generation of intramedullary nails like the Proximal Femoral Nails ${ }^{[8]}$. But these are not found to be very suitable in Indian population because of variation in anthropometry of proximal femur. This may lead to an increased difficulty in placement of femoral neck screws. Therefore, Trochanteric Fixation Nail (TFN) which is smaller in size than Proximal Femoral Nail (PFN) was introduced and has been found suitable for Indian population ${ }^{[9]}$. We have hereby compared the results of use of TFN and hemiarthroplasty in an unstable intertrochanteric fracture.

\section{Materials and Methods}

Our study was a series of 50 cases of unstable intertrochanteric fractures of femur in elderly ( $>65$ years of age) treated with cemented bipolar hemiarthroplasty and TFN. As in this study patients age were more than 65 years and there were more prevalence of osteoporosis in this age group so we included only cemented bipolar hemiarthroplasty. This study was a prospective study conducted over a period of 2 years i.e., from Dec 2014 to Dec 2016. Results were evaluated by Harris Hip Scoring System ${ }^{[10]}$.

\section{Exclusion Criteria}

- Patient with age group less than 65

- Patients with associated injuries that might significantly affect the final functional outcome

- Patients with acetabular changes

- Patient with compound fractures

- Patient with pathological fractures

- Patient with polytrauma

\section{Operative Protocol \\ For Bipolar}

All patients were operated by Antero Lateral approach by coxofemoral bypass. Adequate anteroposterior (AP) radiographs of the hip and proximal femur were taken in full internal rotation to plan for the procedure and pre-operative templating was done.

Supine static quadriceps exercises and ankle pumps are started on day of surgery and sitting quadriceps exercises started on 2nd day postoperatively. Full weight bearing started between Day3-Day 8 postoperatively. Follow up was done post operatively at 1 months, 3 months, 6 months. At each follow up visit patients were evaluated radiologically and clinically. Functional outcome was evaluated by Harris hip score. Radiographs were taken at each follow up to analyzed any evidence of dislocation or prosthesis loosening. Rehabilitation was continued up to 1 year in the form of lifestyle modification like avoiding squatting, sitting cross legged and gait training.

\section{Operative Protocol}

\section{For TFN}

Patient was supine on fracture table. After satisfactory reduction TFN was introduced by greater trochanteric entry. Proximal and distal screws were put through mini incisions.
Postoperatively limb elevation was given. Foot end elevation was given for one day, DVT prophylaxis was given if high risk patient. Static exercise in bed for glutei, hamstrings, quadriceps and breathing exercises were started next day of surgery. Sitting was allowed on next day of surgery with passive exercises in bed. The protocol for weight bearing in unstable fractures: Partial weight bearing after 6 weeks and full weight bearing was started after 3 months approximately. However weight bearing was modified as per the type of fracture, stability of the internal fixation, the fracture union and the tolerance of the patient.

Follow up was done at 1, 3 and 6 month after the surgery. The patients were assessed functionally on the basis of Harris Hip Score and radiologically in the form of anteroposterior and lateral X-rays of the operated hip. Union was decided on the basis of obliteration of fracture line with bridging callus so as to allow unprotected function of the limb.

\section{Results}

Our study had 50 cases of unstable intertrochanteric femur fracture. Out of which 25 patients were operated by cemented bipolar hemiarthroplasty and other 25 patients were operated by TFN.

The following observations were made:

\section{Time taken for surgery}

Table 1:

\begin{tabular}{|c|c|c|c|c|}
\hline $\begin{array}{c}\text { Surgery } \\
\text { Duration(Min) }\end{array}$ & Mean & SD & P Value & Inference \\
\hline Hemiarthroplasty & 82.2 & 10.71 & \multirow{2}{*}{0.3613} & Non-Significant \\
\hline TFN & 78.68 & 15.81 & & \\
\hline
\end{tabular}

There was no significant difference between time taken for TFN and Bipolar hemiarthroplasty.

\section{Time For Full Weight Bearing (FWD)}

Patients of hemiarthroplasty were encouraged for full weight bearing on second day of surgery. They were asked to walk using support of a walker for first few days and then gradually walked with a stick. Most patients walked without any support by fourth day of surgery.

Patients of TFN were partial weight bearing after 6 weeks and full weight bearing was started after 3 months approximately

Table 2

\begin{tabular}{|c|c|c|c|c|}
\hline FWB (Days) & $\begin{array}{c}\text { Mean } \\
\text { (Days) }\end{array}$ & SD & P Value & Inference \\
\hline Hemiarthroplasty & 4.84 & 2.32 & \multirow{2}{*}{0.0001} & Significant \\
\hline TFN & 96.6 & 18.29 & \\
\hline
\end{tabular}

\section{Functional Outcome by Harris Hip Score}

Table 3: Harris Hip Score after 1 Month

\begin{tabular}{|c|c|c|c|c|}
\hline HHS & $\begin{array}{c}\text { Cases } \\
\text { (Hemiarthroplasty })\end{array}$ & $\boldsymbol{\%}$ & $\begin{array}{c}\text { Cases } \\
\text { (TFN) }\end{array}$ & $\boldsymbol{\%}$ \\
\hline $90-100$ (Excellent) & 1 & 4 & 0 & 0 \\
\hline $80-89$ (Good) & 12 & 48 & 0 & 0 \\
\hline $70-79$ (Fair) & 11 & 44 & 6 & 24 \\
\hline$<70$ (Poor) & 1 & 4 & 19 & 76 \\
\hline Total & 25 & 100 & 25 & 100 \\
\hline
\end{tabular}


Table 4: Average Harris Hip Score after 1 Month

\begin{tabular}{|c|c|c|c|c|}
\hline HHS After 1 Month & Average & Sd & P Value & Inference \\
\hline Hemiarthroplasty & 79.92 & 6.23 & \multirow{2}{*}{$<0.0001$} & \multirow{2}{*}{ Significant } \\
\hline Tfn & 66.96 & 5.29 & & \\
\hline
\end{tabular}

After 1 month results were statistically more significant.

Table 5: HHS after 6 Months

\begin{tabular}{|c|c|c|c|c|}
\hline HHS & Cases (Hemiarthroplasty ) & $\mathbf{\%}$ & Cases (TFN) & $\mathbf{\%}$ \\
\hline $90-100$ (Excellent) & 3 & 12 & 1 & 4 \\
\hline $80-89$ (Good) & 12 & 48 & 8 & 32 \\
\hline $70-79$ (Fair) & 9 & 36 & 10 & 40 \\
\hline$<70$ (Poor) & 1 & 4 & 6 & 24 \\
\hline TOTAL & 25 & 100 & 25 & 100 \\
\hline
\end{tabular}

Table 6: Average HHS after 6 Months

\begin{tabular}{|c|c|c|c|c|}
\hline HHS After 6 Month & Average & SD & P Value & Inference \\
\hline Hemiarthroplasty & 81.72 & 6.74 & \multirow{2}{*}{0.0002} & \multirow{2}{*}{ Significant } \\
\hline TFN & 73.2 & 8.19 & & \\
\hline
\end{tabular}

After 6 months also results were statistically significant.

\section{Complications}

Table 7

\begin{tabular}{|c|c|c|c|c|c|c|c|}
\hline Complications & No. of Patients (Hemiarthroplasty) & $\mathbf{\%}$ & No of Patients (TFN) & $\mathbf{\%}$ & Z Score & P Value & Inference \\
\hline Pulmonary Embolism & 00 & $0 \%$ & 01 & $4 \%$ & 1.01 & 0.31 & Not Significant \\
\hline Superficial infection & 02 & $8 \%$ & 01 & $4 \%$ & 0.59 & 0.54 & Not Significant \\
\hline Deep vein thrombosis & 01 & $04 \%$ & 04 & $16 \%$ & 1.41 & 0.51 & Not Significant \\
\hline Dislocation & 01 & $4 \%$ & 00 & $0 \%$ & 1.01 & 0.31 & Not Significant \\
\hline Pressure sore & 01 & $4 \%$ & 06 & $24 \%$ & 2.03 & 0.04 & Significant \\
\hline Limb shortening & 01 & $4 \%$ & 06 & $24 \%$ & 2.03 & 0.04 & Significant \\
\hline Implant breakage & 00 & $0 \%$ & 01 & $4 \%$ & 1.01 & 0.31 & Not Significant \\
\hline Screw cut out & 00 & $0 \%$ & 01 & $4 \%$ & 1.01 & 0.31 & Not Significant \\
\hline
\end{tabular}

Four case of TFN and one case of hemiarthroplasty had DVT which was managed by close monitoring and conservative management in ICU setup. Two cases of hemiarthroplasty and one case of TFN had superficial infection which was managed by targeted antibiotic therapy after culture and sensitivity testing. One case of hemiarthroplasty had pre-operative pressure sore which was managed by aggressive nursing care and since the patient had early post-operative mobilization, it helped in early healing of the wound. Six case of TFN had pressure sore due to prolonged recumbancy. One patient of hemiarthroplasty had limb shortening of $1 \mathrm{~cm}$ which was managed by shoe raises and four patients of TFN had $1 \mathrm{~cm}$ limb shortening and two had $1.5 \mathrm{~cm}$ limb shortening due to varus collapse. One case of hemiarthroplasty had postoperative postero-superior dislocation, which occurred on fourth post-operative day due to excessive hip flexion by the patient, which was managed by closed reduction under anaesthesia. The limb was kept in Thomas splint for three weeks post which delayed mobilization was started.

One patient of TFN had pulmonary embolism which was managed by intensive care in ICU setup. One patient of TFN had implant breakageand one had screw cut out which need further operation.

So in TFN there was significant complication like pressure sore and DVT due to prolonged immobilization.

\section{Discussion}

The treatment of intertrochanteric fracture is still associated with some failures. High stress concentration that is subject to multiple deforming forces and high incidence of complications reported after surgical treatment compels the surgeon to give a second thought regarding selection of proper implant. A large number of fixation implants has been devised and discarded. The treatment still merits the type of fracture and condition of the patient.

Early ambulation following surgeries is important for preventing complications that can be caused by long term bed rest in elderly patients with poor general conditions. The poor mechanical properties of the weak and osteoporotic bones in elderly patients do not provide a good purchase for the screws, which subsequently lead to an early biomechanical failure. Studies with Trochanteric femoral nail (TFN) in unstable inter-trochanteric fractures have shown a high incidence of complications. Largest series available in literature comparing both methods is study done by Peifu Tang et al ${ }^{[11]}$ with 303 cases. He reported complications which include infection, non union, screw breakage, dislocations and screw cut outs. Other studies include studies done by Jun Shen et $a l^{[12]}$ and Kim et al ${ }^{[13]}$ with 124 and 58 patients respectively.

So for internal fixation early mobilization is better to be avoided in cases with comminution, osteoporosis, or poor screw fixation ${ }^{[14,15]}$.

Primary hemiarthroplasty offers a modality of treatment that provides adequate fixation and early mobilization in these patients thus preventing postoperative complications such as pressure sores, pneumonia, atelectasis and pseudoarthrosis ${ }^{[16]}$. 


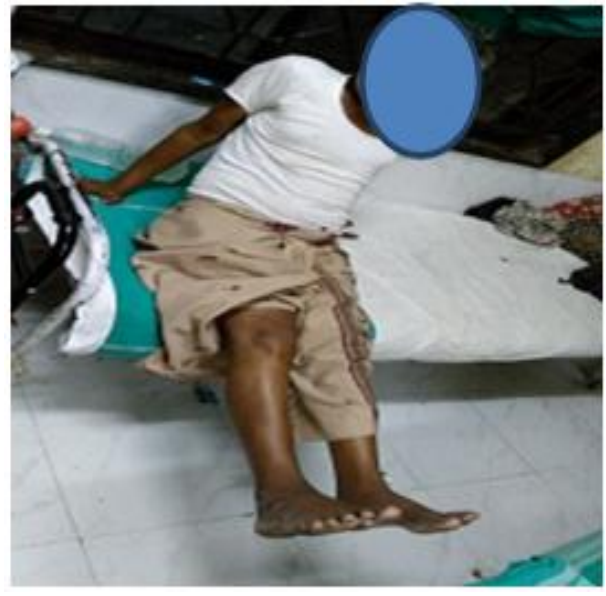

(1)

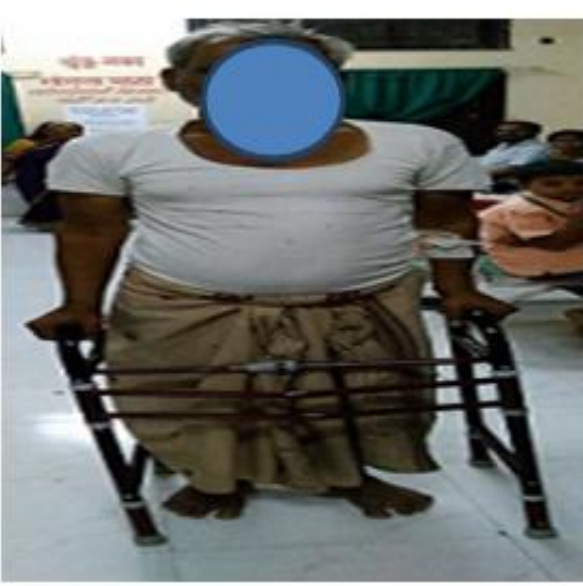

(2)

Image 1 2: Patient mobilised with sitting quadriceps exercises and full weight bearing on $2^{\text {nd }}$ post op day

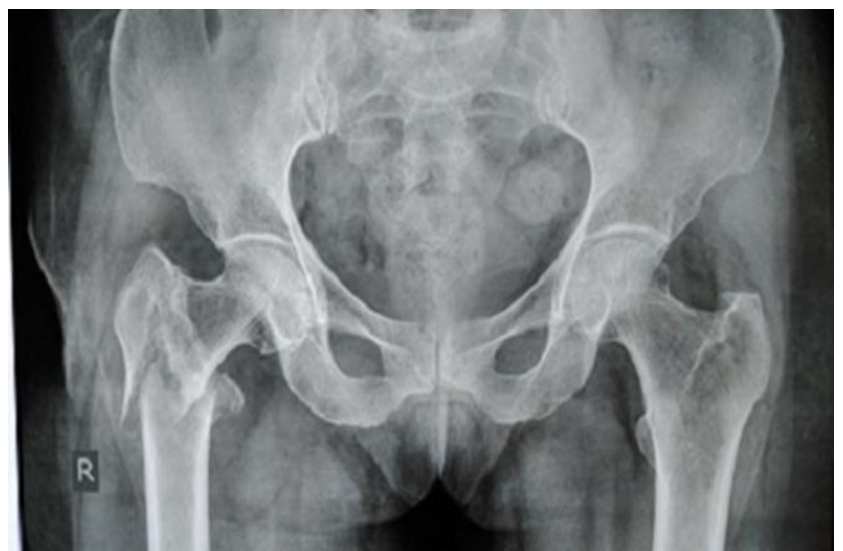

Image 3: Pre op X ray

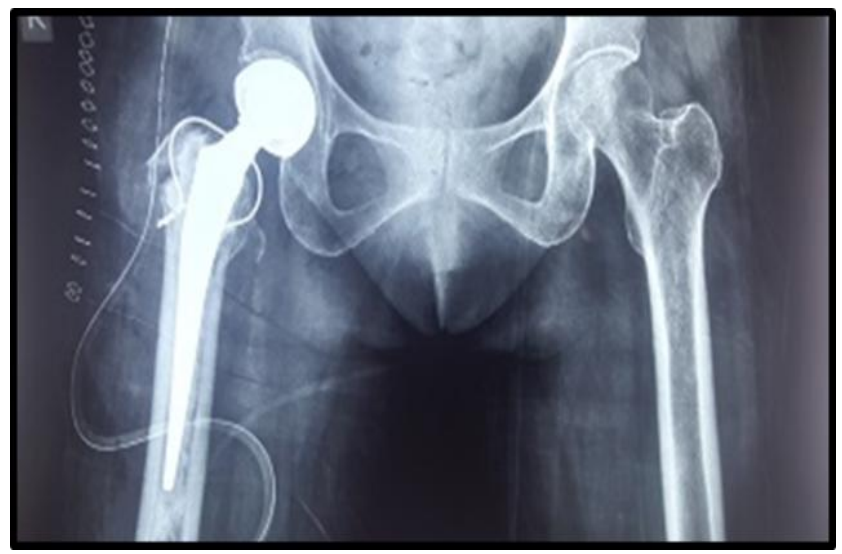

Image 4: After Bipolar hemiarthroplasty

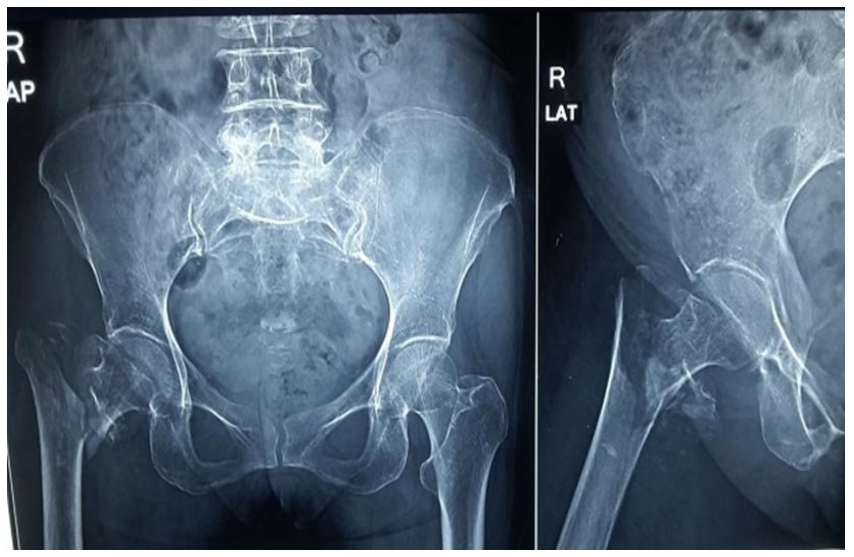

Image 5: Pre op X ray

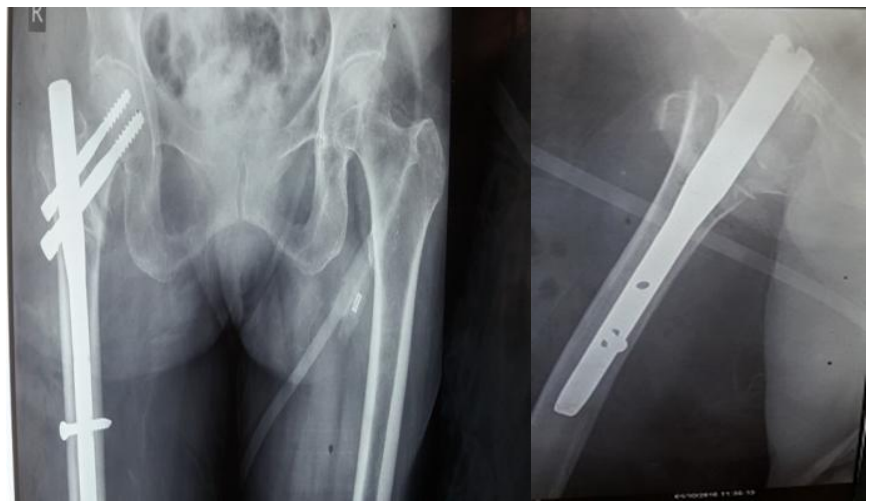

Image 6: After Trochantric Femoral Nailing

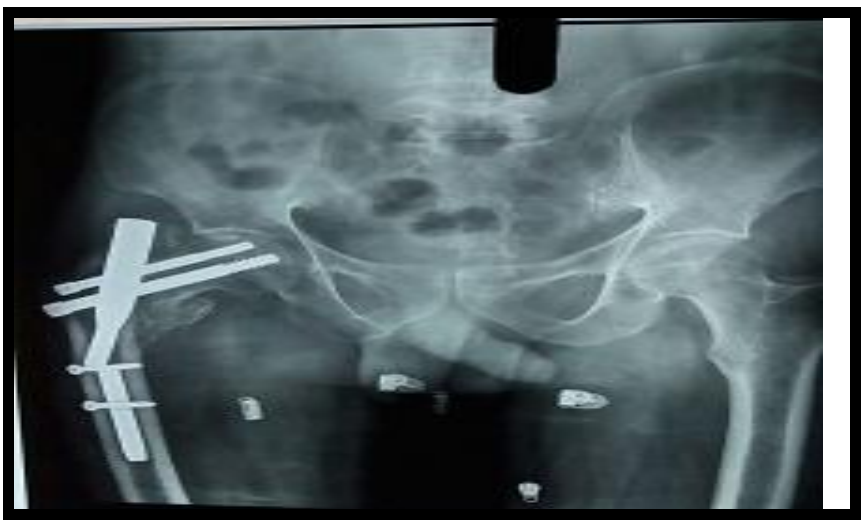

Image 7: Implant Failure and varus collapse

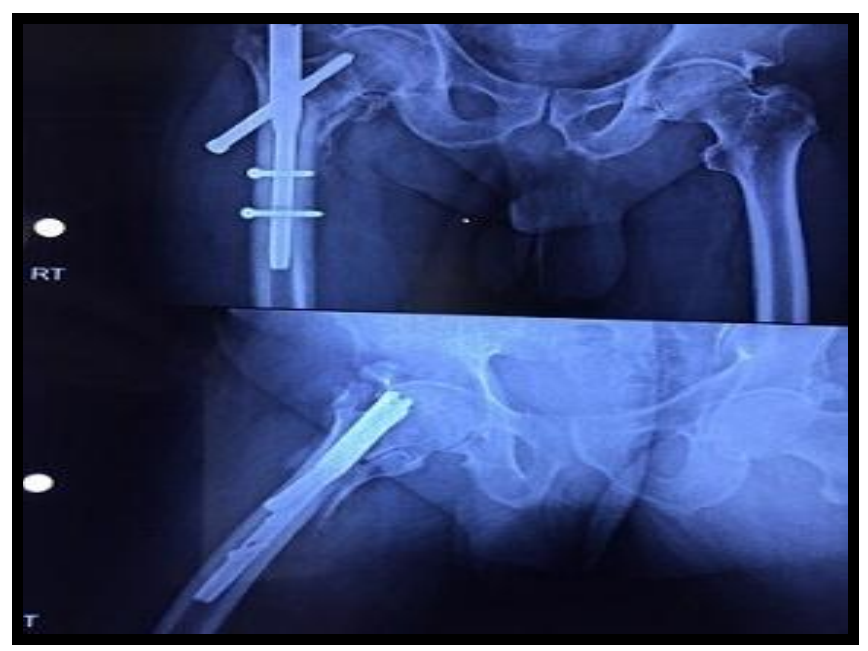

Image 8: Screw back out 


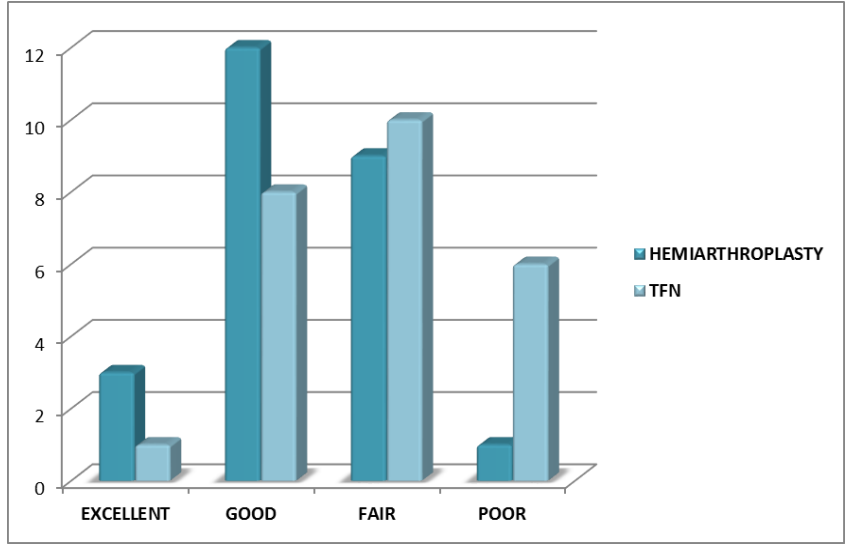

\section{Conclusion}

- The cornerstone of management of such fractures is early surgery, followed by mobilization. Early mobilization is very essential, particularly in patients with other medical co-morbidities and also to prevent post-operative complications.

- Hence Cemented bipolar hemiarthroplasty offers a modality of treatment that provides adequate fixation and early mobilization in these patients thus preventing postoperative complications such as pressure sores, chest infection and DVT, as compared to TFN where there was more chance of complication due to prolonged immobilization. So Bipolar Hemiarthroplasty will have a direct effect on the general condition and the postoperative rehabilitation.

- This modality overcomes the problems faced with internal fixation (TFN) for treating unstable fractures like head perforations, implant breakage, screw cut out.

Thus, cemented bipolar hemiarthroplasty should be considered as one of the best modalities for the primary treatment of comminuted unstable intertrochanteric fractures of femur in elderly as compared to TFN.

\section{References}

1. Koval KJ, Aharonoff GB, Rokito AS. Patients with femoral neck and intertrochanteric fractures. Are they the same? Clin Orthop Relat Res. 1996; 330:166-172.

2. Campbell's operative orthopedics; Philadelphia: Mosby Elsevier; 2008; 11:3239-3256.

3. Eiskjaer S, Otsgard SE, Jakobsen BW, Jensen J, Lucht U. Years of potential lost after hip fracture among postmenopausal women. Actaorthop Scand. 1992; 63(3):293-296.

4. Dahl E. Mortality and life expectancy after hip fractures. 1 Actaorthop Scand. 1980; 51(1):163-170.

5. Kim WY, Han CH, Park JI, Kim JY. Failure of intertrochanteric fracture fixation with a dynamic hip screw in relation to pre-operative fracture stability and osteoporosis. Intorthop. 2001; 25:360-2.

6. Karsson S. Treatment of osteoporotic fractures. Scand J Surg. 2002; 91:140-6.

7. Jensen JS, Tondenvold E, Mossing N. Comminuted trochanteric fractures treated with the sliding screw plate system: A biochemical study of comminuted trochanteric fractures. III, ActaOrthop Scand. 1978; 49:392-7.

8. Meislin RJ, Zuckerman JD, Kummer FJ. Abiomechanical evaluation of the gamma nail. J Bone Joint Surg. 1998; 70-A:239.
9. Anchal Kumar. Femoral neck geometry and rationale of placement of the proximal femoral nail and its relationwith fracture risk in the Indian population review of 120cases. 2010: Gulmohar Hospital, Ranchi

10. Harris H.: Harris Hip Score, Journal of Bone and Joint Surgery American. 1969; 51-A(4):737-55.

11. Peifu Tang. Proximal femoral nail antirotation versus hemiarthroplasty: A study for treatment of intertrochanteric fractures. Injury. 2012; 43(6):876-881.

12. Jun Shen. Bipolar hemiarthroplasty compared with internal fixation for unstable intertrochanteric fractures in elderly patients. J Orthop Science. 2012; 17(6):722-9.

13. Kim SY, Kim YG, Hwang JK. Hemiarthroplasty compared with intramedullary fixation of unstable intertrochanteric fractures: J Bone Joint Surg. 2005; 87A:2186-92.

14. Wolfgang GL, Bryant MH, O’Neill JP. Treatment of intertrochanteric fracture of the femur using sliding screw plate fixation. Clin Orthop Relat Res. 1982; 163:148-58. [PubMed]

15. Sernbo I, Johnell O, Gentz CF, Nilsson JA. Unstable intertrochanteric fractures of the hip: Treatment with Ender pins compared with a compression hip-screw. J Bone Joint Surg Am. 1988; 70:1297-303. [PubMed]

16. Haentjens P, Casteleyn PP, DeBoeck H Treatment of comminuted intertrochanteric and subtrochanteric fractures in elderly patients: primary bipolar arthroplasty compared with internal fixation. J Bone Joint Surg. 1989; 71A:1214-1255. 